\title{
Validation of a membrane touch biosensor for the qualitative detection of IgG class antibodies to herpes simplex virus type 2
}

Tony Loughman

Aalto Bio Reagents Ltd

Baljit Singh

Technological University Dublin, baljit.singh@tudublin.ie

Brian Seddon

Technological University Dublin

See next page for additional authors

Follow this and additional works at: https://arrow.tudublin.ie/ittsciart

Part of the Medicinal-Pharmaceutical Chemistry Commons, and the Medicine and Health Sciences Commons

\section{Recommended Citation}

Loughman T, Singh B, Seddon B, Noone P, Santhosh P. Validation of a membrane touch biosensor for the qualitative detection of IgG class antibodies to herpes simplex virus type 2. Analyst. $2017 \mathrm{Jul}$ 24;142(15):2725-2734. doi: 10.1039/c7an00666g. PMID: 28708188.

This Article is brought to you for free and open access by the School of Science and Computing at ARROW@TU Dublin. It has been accepted for inclusion in Articles by an authorized administrator of ARROW@TU Dublin. For more information, please contact arrow.admin@tudublin.ie, aisling.coyne@tudublin.ie, gerard.connolly@tudublin.ie.

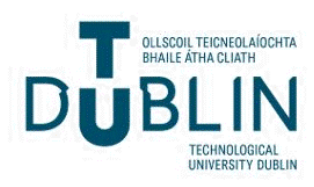




\section{Authors}

Tony Loughman, Baljit Singh, Brian Seddon, Philip Noone, and Padmanabhan Santhosh 


\title{
Analyst
}

\section{PAPER}

View Article Online

View Journal | View Issue
(A) Check for updates

Cite this: Analyst, 2017, 142, 2725

\section{Validation of a membrane touch biosensor for the qualitative detection of IgG class antibodies to herpes simplex virus type 2}

\author{
Tony Loughman, ${ }^{\mathrm{a}}$ Baljit Singh, (id ${ }^{\mathrm{b}}$ Brian Seddon, (D) *b Philip Noone ${ }^{\mathrm{a}}$ and \\ Padmanabhan Santhosh ${ }^{b}$
}

\begin{abstract}
A novel type of biosensor was assessed for application to the qualitative determination of circulating antibodies to herpes simplex virus type 2 (HSV-2). The device utilises a high activity HSV-2 type specific gG2 antigen for antibody capture and commercially available ELISA reagents. The study compares the diagnostic performance of a prototype HSV-2 biochip to well-established in vitro tests routinely applied in clinical procedures. A panel of human serum samples $(n=60)$ previously characterised for HSV-2 serological status using the DiaSorin LIAISON ${ }^{\circ}$ HSV-2 chemiluminescent immunoassay were assayed on the HSV-2 biochip and the Focus Diagnostics HerpeSelect ${ }^{\circledR} 2$ ELISA IgG kit to determine concordance with the predicate test method. Sensitivity and specificity of the HSV-2 biochip were found comparable to both the DiaSorin and Focus test methods. Sample index values calculated from the immunoassay response of the biochip's coulometric sensors indicated a high degree of linear correlation of the dataset with the corresponding index values from the DiaSorin LIAISON® test $\left(r^{2}\right.$ 0.8799) and Focus HerpeSelect ${ }^{\circledR}$ test $\left(r^{2}\right.$ 0.8794). The HSV-2 biochip demonstrated excellent diagnostic performance in qualitative and semi-quantitative measurements, matching closely the performance of two diagnostic industry standard predicate methods.
\end{abstract}

Received 21st April 2017

Accepted 28th June 2017

DOI: $10.1039 / \mathrm{c} 7 \mathrm{an} 00666 \mathrm{~g}$

rsc.li/analyst
HSV-2 are asymptomatic and have no reported history of genital lesions. ${ }^{10,11}$ Consequently, most HSV-2 transmission occurs from those that are not aware that they are infected. ${ }^{1,12}$

The classical gold-standard for genital HSV diagnosis in the laboratory has been viral culture followed by species typing to determine if the causative agent is HSV-1 or HSV-2. ${ }^{13}$ In asymptomatic individuals, serological testing can be used to determine previous HSV infection. HSV-1 and HSV-2 share significant identity with up to $83 \%$ sequence homology observed in the protein-coding regions of their genomes. ${ }^{14}$ Due to the significant similarities between HSV types, serological distinction between them is primarily reliant on the immune reaction to glycoprotein G. ${ }^{15-17}$ Accurate serological tests are based on high purity glycoprotein G-1 (gG1) and glycoprotein G-2 (gG2) antigens for HSV-1 and HSV-2 respectively. ${ }^{18}$ A number of such tests are commercially available. The Focus Diagnostics HerpeSelect $\circledast 2$ ELISA IgG test utilises a recombinant gG2 antigen and is approved by the Food and Drug Administration (FDA) for use in sexually active adults and expectant mothers. This test has been widely used in various studies and patient populations. ${ }^{19-23}$ A constraint of contemporary HSV-2 serological tests in routine clinical use is the requirement of specialised accessory equipment and indeed highly skilled technicians for their operation. It can often be difficult to access

\footnotetext{
${ }^{a}$ Aalto Bio Reagents Ltd, Church Lane, Rathfarnham Village, Dublin 14, Ireland ${ }^{b}$ MiCRA Biodiagnostics Technology Gateway, Synergy Centre - CASH Building, Institute of Technology Tallaght, Dublin 24, Ireland.

E-mail: brian.seddon@ittdublin.ie, Baljit.Singh@ittdublin.ie
} 
these tests in resource-limited settings for example in developing countries. Therefore a current clinical need exists for an easy-to-use and reliable, cost-effective serological test for HSV-2 diagnosis.

Today the majority of HSV-2 serological testing is performed using optical or chemiluminescent enzyme immunoassays in diagnostic laboratories due to the requirement for specialised equipment and trained assay technicians. It would be preferable to move towards point-of-care (PoC)-style HSV-2 serological tests, the primary benefit being the rapid sampleto-answer time of minutes as opposed to hours. This permits immediate preliminary diagnosis in a doctor's office or STD clinic during patient visits. Additionally HSV-2 PoC tests could be used in resource-limited settings where HSV-2 is prevalent and there is no access to centralised diagnostic laboratory services. There are PoC tests available for the qualitative determination of circulating antibodies against HSV-2, such as the Uni-GoldTM $^{\mathrm{TM}}$ HSV-2 Rapid Test or the Sure-Vue ${ }^{\circledR}$ HSV-2 Rapid Test. These tests utilise colloidal gold conjugates to detect bound anti-HSV-2 IgG. The formation of a coloured line or spot at a test region containing gG2 antigen determines whether the sample is positive or negative. Assays such as these are potentially subject to operator variability since they rely on visual assessment of the test area: a particular problem in cases of samples that may be borderline or low positive. By contrast, a potentiostat-based electronic reader for the HSV-2 biochip test, measures, calculates and displays a sample index number for HSV-2 leaving little ambiguity on the HSV-2 serological status of a test sample.
Under consideration in this work is an intriguing type of microfluidic biosensor technology, i.e. membrane-touch biochip, which offers promise as a portable instrument for out-of-laboratory diagnostic tests. The device is engineered as a flow-through microfluidic system composed of a reaction chamber linked to series coulometric sensors. The reaction chamber supports immunochemical reagents, e.g. an antigen, and can be integrated with any exterior liquidhandling device for the delivery of sample and immunoassay reagents. Coulometric sensors on the chip are electronically activated at the end of an assay sequence by an on-chip micro-hydraulic mechanism actuated by a membrane-touch pump. ${ }^{24}$ The sensors are responsive to redox molecules emanating from peroxidase activity of the immunoassay. The chip's detection operates by a millivoltcoulometry technique, whereby a small potential difference is applied across two indicator electrodes of a sensor and an electrochemical charge measured by a potentiostat instrument.

The utility of the membrane-touch biochip as a reliable immunoassay device was demonstrated in HSV-2 antibody measurements using a high purity recombinant gG2 protein to functionalise the biochip reaction chamber. The analytical performance of this HSV-2 biochip was assessed in terms of assay sensitivity and specificity against FDA-cleared, market leading HSV-2 serological tests from DiaSorin (LIAISON® HSV-2 IgG) and Focus Diagnostics (HerpeSelect ${ }^{\circledR} 2$ ELISA IgG). The study's findings and consequences for the biochip design as a practical immunoassay sensor are discussed.
Dr Tony Loughman received his PhD in Molecular Microbiology from Trinity College Dublin in 2005 studying the molecular interaction between bacterial surface proteins and host cell receptors. He has over 10 years' experience in the in vitro diagnostic industry in the design \& development of ELISA assays for biomarkers of kidney damage and in development and validation of molecular diagnostics for various cancer biomarkers.

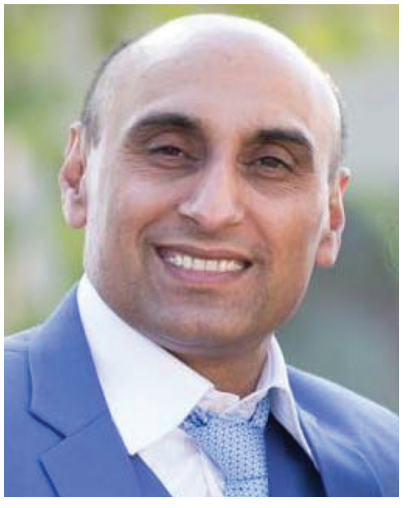

Baljit Singh
Baljit Singh is working as Project Manager (Business Development) at MiCRA Biodiagnostics. His responsibilities include research and business development, delivery of industry projects, technical reports, grant/funding applications, centre development and IP commercialisation. He was awarded Doctorate (nanoscaled materials and electrochemistry) from ITT Dublin and M. Tech (Advanced Chemical Analysis) from Indian Institute of Technology-Roorkee. He has around 25 research publications and 1 patent on sensors and microfluidic-diagnostic systems.

Expertise: Advancement of chemical \& bio-sensors technologies for human/animal diagnostics, dairy/food and water testing. Biomaterials fabrication and immunoassay techniques. Sensors for food allergens, contaminants/residues and rapid microbiology analysis. Carbon supported metallic nanocomposites, electroactive and nanoporous materials, plastic thin-film devices and printed electrode systems. 


\section{Experimental}

Materials and methods

Components of the membrane-touch biochip were fabricated from plastic-film laminates based on proprietary polyester materials (Melinex, DuPont Teijin Films, USA). Coulometric sensors were patterned onto the chip by a screen-printing process (DEK248 semi-automatic screen printer, UK), utilising biosensor-grade carbon conducting ink (DuPont, USA). Microfluidic features were designed using CorelDraw drawing software and component parts machined by a $\mathrm{CO}_{2}$ laser (Epilog Zing, USA). The base polymer chip was functionalised with a recombinant gG2 antigen (Aalto Bio Reagents, Ireland) diluted to $5 \mu \mathrm{g} \mathrm{mL} \mathrm{m}^{-1}$ in $50 \mathrm{mM}$ carbonate buffer, $\mathrm{pH} 9.6$ and $15 \mu \mathrm{L}$ solution (containing $75 \mathrm{ng}$ gG2 antigen) and injected into the reaction chamber. Chips were incubated at ambient temperature $\left(20-25^{\circ} \mathrm{C}\right)$ for 90 minutes to allow adsorption of the gG2 antigen to the reaction chamber surface, see Fig. 1 and 2. The gG2 solution was then aspirated and the biochip reaction chamber washed by injection of $50 \mu \mathrm{L}$ phosphate buffered saline, pH 7.4 containing $0.05 \%$ (v/v) Tween 20 (PBST buffer). Wash buffer was completely aspirated from the reaction chamber and $15 \mu \mathrm{L}$ of $1 \%(\mathrm{w} / \mathrm{v})$ bovine serum albumin (BSA) in $50 \mathrm{mM}$ carbonate buffer, $\mathrm{pH} 9.6$ was injected to block potential non-specific binding sites on the reaction chamber surface. Following a 90 minutes incubation at ambient temperature $\left(20-25^{\circ} \mathrm{C}\right)$, the BSA solution was aspirated and the biochips were dried by incubation at $37^{\circ} \mathrm{C}$ for 60 minutes. HSV-2 gG2 coated biochips were stored at $2-8{ }^{\circ} \mathrm{C}$ until required for immunoassay.

\section{HSV-2 immunoassay with biochip}

The HSV-2 biochip assay was performed using commercially available ELISA reagents from the HerpeSelect ${ }^{\circledR} 2$ IgG kit (Focus Diagnostics, CA, USA; product EL0920G). Control and serum samples were mixed thoroughly using a vortex mixer and diluted 51-fold in sample diluent. Diluted samples $(15 \mu \mathrm{L})$ were injected into gG2 antigen-coated biochip reaction chambers and incubated at ambient temperature $\left(20-25{ }^{\circ} \mathrm{C}\right)$

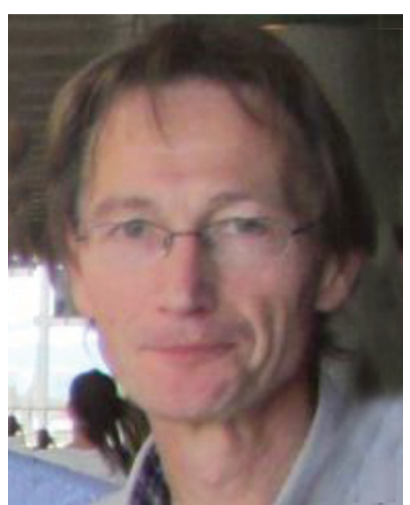

Brian Seddon
Brian Seddon is project scientist at MiCRA Biodiagnostics, an industry focused research centre located at Tallaght, Dublin. His role concerns the technical and commercial advancement of IVD technologies and in-line process sensors. He specialises in the design and engineering of electrode systems for applications to biosensors and instrumentation for chemical and micro-organism detection.
Philip Noone is the managing director of Aalto Bio Reagents, Dublin, Ireland.

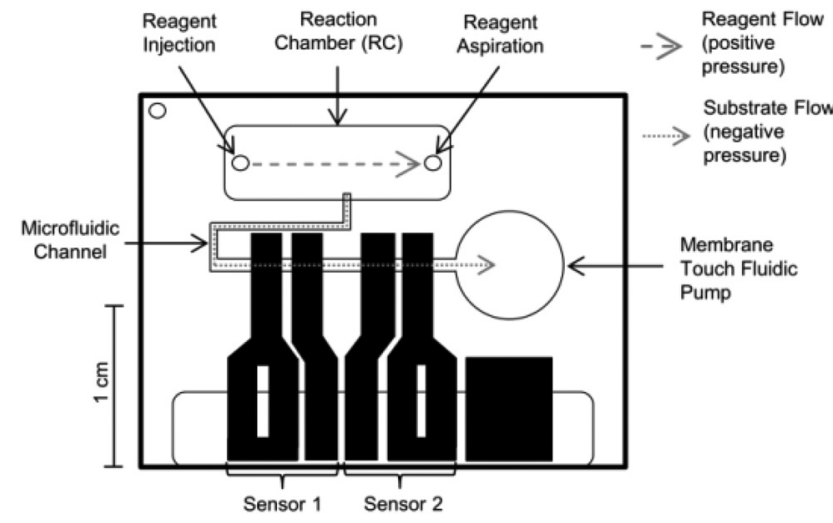

Fig. 1 A schematic representation of the biochip platform is shown. The reaction chamber capacity is $15 \mu \mathrm{L}$. Assay reagents are injected into the reaction chamber using a pipette, incubated for the required time to allow binding to occur, and aspirated before subsequent injection of the next reagent. The reaction chamber contents do not enter the microfluidic channel leading to the sensors during the immunoassay routine. Prior to injection of the substrate solution to indirectly detect the analyte of interest (via the captured enzyme conjugate), the membranetouch device is engaged by application of a force. Upon incubation of the substrate solution in the reaction chamber, the force is removed from the membrane-touch pump; this creates negative pressure which draws the reaction chamber contents (i.e. the electrochemically active substrate solution) into the microfluidic channel and into contact with the sensors. The electrochemical charge is measured at the electrodes by millivolt coulometry.

for 30 minutes to allow adsorption of specific anti-gG2 antibody in the sample to the functionalised reaction chamber surface. The sample was then aspirated and the reaction chamber washed twice with $50 \mu \mathrm{L}$ PBST buffer per wash. Goatanti-human IgG-peroxidase conjugate supplied with the ELISA kit was diluted 3 -fold in $1 \%(\mathrm{w} / \mathrm{v})$ BSA in PBST and $15 \mu \mathrm{L}$ of diluted conjugate was injected into the washed biochip reaction chamber. The conjugate was incubated for 30 minutes at ambient temperature $\left(20-25^{\circ} \mathrm{C}\right)$ to allow binding to captured anti-gG2 antibody from the test sample. The conjugate was then aspirated and the reaction chamber washed twice with $50 \mu \mathrm{L}$ PBST buffer per wash. Remaining wash buffer within the 


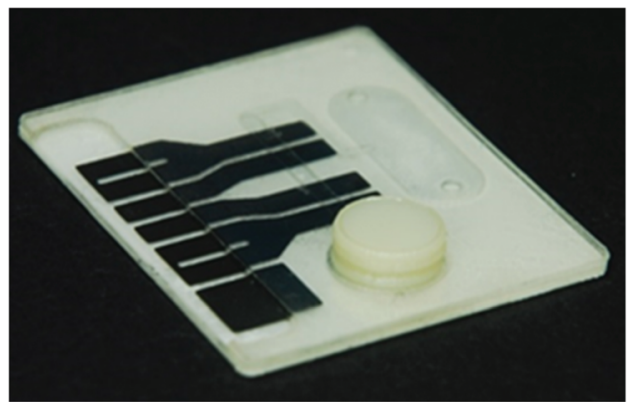

Fig. 2 Photograph of the generic membrane-touch biochip used for the HSV-2 ELISA. Chip dimensions: $25 \mathrm{~mm} \times 30 \mathrm{~mm}$. Reaction chamber $5 \mathrm{~mm} \times 14 \mathrm{~mm}$ and membrane-touch pump $7 \mathrm{~mm}$ diameter. The indicator electrodes of sensors 1 and 2 have an area of $2 \mathrm{~mm}^{2}$. The membrane-touch pump can be seen in the foreground of the image as a button elevated from the chip's base.

reaction chamber was aspirated and the membrane-touch device engaged by applying an external force. A volume of $15 \mu \mathrm{L}$ of peroxidase substrate solution, $3^{\prime} 3^{\prime} 5^{\prime} 5^{\prime}$-tetramethylbenzidine (TMB), was then injected into the reaction chamber to detect the captured peroxidase conjugate. Following 5 minutes incubation, the applied force was removed from the membrane-touch device to create negative pressure. This action filled the microfluidic channel (shunt) leading to the sensors with reacted TMB substrate. Sensors were then activated by a $50 \mathrm{mV}$ potential difference from a CH660 potentiostat (CH Instruments Inc., TX, USA). The electrochemical charge from the reacted substrate in contact with both sensors was measured by millivolt-coulometry. Coulometric data in immunoassay studies are tabulated in units of microcoulombs $(\mu \mathrm{C})$ : charge is sampled from charge-time measurements at 10 seconds $\left(Q_{10}\right)$ for both sensors on the biochip.

\section{Clinical sample analysis}

A panel of 60 human serum samples previously screened using the LIAISON® HSV-2 Type Specific IgG chemiluminescent immunoassay test (DiaSorin, MN, USA) was purchased from a commercial vendor (Cerba Specimen Services, France) in order to validate the HSV-2 biochip assay. This sample panel comprised 30 positive samples (LIAISON® assay index >1.1), 29 negative samples (LIAISON® assay index $<0.9$ ) and one equivocal sample (LIAISON $\AA$ assay index $=1.0$ ). The samples were collected from a predominantly French population, with 49

Santhosh Padmanabhan received his Ph.D. degree in Industrial Chemistry from Alagappa University, India in 2005. He has conducted research activities at Kyungpook National University (South Korea), Max-Planck-Institute for Solid State Research (Germany), University of California-San Diego (USA), University of Pittsburgh (USA), and MiCRA-Biodiagnostics (Ireland). Currently, he is working as a Senior Scientist in Mouthsense Labs, California, USA. He has published over 63 peer reviewed research articles and is the inventor on 5 patents.
$(81.7 \%)$ collected from mainland France. Nine of the remaining 11 samples (15.0\%) were collected in various French oversea territories (three samples from Mayotte, two samples each from Martinique and French Guiana, one sample each from French Polynesia and Reunion Island). The remaining two samples (3.3\%) were from Madagascar and Morocco. The samples were from a predominantly female population (42 of 60 samples $(70 \%))$. The median age of the entire patient population was 40.5 years with patients ranging in age from 15-85 years. The sample index values on the DiaSorin LIAISON ${ }^{\circledR}$ HSV-2 Type Specific IgG test were supplied by Cerba Specimen Services. No other patient information was supplied. The majority of negative samples were supplied with reported index values of $<0.5$. Each sample was assayed in triplicate on the HSV-2 biochip assay as described above. Strongly reactive samples (LIAISON $®$ index $>4.0$ ) were additionally retested on the HSV-2 biochip at increased sample dilution factors (102-fold or 204-fold). All serum samples were assayed using the Focus Diagnostics HerpeSelect ${ }^{\circledR} 2$ IgG ELISA kit according to the manufacturer's instructions.

\section{Data analysis}

The average electrochemical charge value for test samples and the cut-off control were calculated from the available 6 replicate measurements ( 3 biochips per sample, 2 sensors per biochip). Sample index values were calculated by expressing the mean charge as a ratio of the average charge of the cut-off calibrator sample assayed during the same testing run. Samples with index values $>1.1$ were classified as positive for anti-HSV-2 antibody. Index values for high positive samples were corrected for increased sample dilution factor relative to the cut-off calibrator. Similarly, optical absorbance values for the Focus ELISA assay were converted to index values by expressing the average test sample OD as a ratio of the mean OD of the cut-off calibrator sample assayed during the same testing run.

The cut-off calibrator is a commercially available component of the HerpeSelect 2 IgG ELISA kit (Focus Diagnostics, cat. \# EL0920G). This ELISA test is both CE in vitro diagnostic (IVD) and Food \& Drug Administration approved as an in vitro diagnostic for the qualitative determination of human IgG class antibodies to HSV-2 in human sera and is one of the market leading tests for HSV-2 antibody qualitative determination. Therefore the qualitative index for HSV-2 antibody positivity or negativity is regarded as accurate and extensively applied in immunoanalysis. Moreover, the Focus Diagnostics cut-off calibrator is a human serum matrix formulated to contain a level of anti-HSV-2 antibodies to give the optimum differentiation between negative and positive sera. In designing the assay, Focus validated the cut-off calibrator against a reference lab-developed Western blot method using five serum panels $(n=383)$ tested on both ELISA and Western blot methods. In ELISAs, samples yielding an absorbance measurement $\geq 10 \%$ lower than the cut-off calibrator are defined as HSV-2 antibody negative. Samples yielding an absorbance measurement $\geq 10 \%$ higher than the cut-off calibrator are 
defined as HSV-2 antibody positive. Samples that are within the $\pm 10 \%$ margin of the within-run cut-off calibrator are regarded as equivocal and are either re-tested or the patient is resampled after a period of weeks to re-test the immune response. The same classification for sample positivity or negativity for anti-HSV-2 IgG in the biochip test is utilised; that is if a sample is either $<90 \%$ or $>110 \%$ of the within-day HSV-2 biochip calibrator signal, the sample is classed as negative or positive respectively.

\section{Results and discussion}

\section{Biochip operation}

A schematic illustration and photographic image of the membrane-touch biochip are presented in Fig. 1 and 2. Biochip architecture includes a micro-volume flow-through reaction chamber with a t-connector fluidic link to two series coulometric sensors. A membrane-touch pump communicates liquid held in the reaction chamber to the sensors via a microfluidic shunt channel. The sensors themselves consist of an anode and cathode pair, which are operated by the application of a millivolt potential difference. In this study, the surface of the reaction chamber is functionalised with recombinant HSV-2 gG2 protein in order to selectively capture anti-HSV-2 antibodies contained within test samples. Control or serum samples are diluted in assay diluent and injected into the gG2 reaction chamber and incubated to allow binding of IgG in the test sample to the reaction chamber surface. Bound IgG is detected by subsequent injection and incubation of an antihuman IgG antibody-peroxidase conjugate. The horseradish peroxidase (HRP) label is measured through its oxidation of the substrate TMB in the presence of hydrogen peroxide. The rate of oxidation of TMB is proportional to the surface bound peroxidase and thus to the amount of anti-gG2 IgG captured from the original test sample. Peroxidase-mediated oxidation of TMB in the presence of hydrogen peroxide yields a blue coloured product which is generated by way of a two-electron reaction mechanism. ${ }^{25}$ The electron release in the enzyme oxidation reaction can be measured by polarised electrodes and therefore by the printed electronic sensors on the biochip. It should be realise that the entire fluidic sequence of the ELISA takes place only within the reaction chamber of the biochip and not at the coulometric sensors.

The isolation of the sensors on biochip from the reaction chamber is a significant design improvement, having the effect of reducing background interferences common to printed immunoelectrodes. At the stage where the substrate is injected onto the biochip, the membrane-touch microfluidic is engaged by the application of an external force. After a sufficient substrate-reaction time, this force is removed from the membrane-touch device creating a negative pressure which diverts oxidised TMB substrate into a shunt channel making contact to the sensors.

The electrochemical charge is measured once a small potential difference is applied across an electrode pair - a tech- nique referred to as potential-step coulometry. For analysis of HSV-2 biochip performance, electrochemical charge values were sampled at 10 seconds $\left(Q_{10}\right)$ from charge-time plots.

One aspect of the sensor response which should be recognised is the parabolic dependence of $Q_{10}$ on peroxidase concentration. This is shown in eqn (1). An equation adapted for coulometric peroxidase electrodes from the original work of Kies on a theoretical approximation for dead-stop end-point amperometry. ${ }^{26}$

$$
\begin{gathered}
Q=k \cdot\left[1 \pm \sqrt{1-4\left(\frac{\Delta-1}{\Delta+1}\right)^{2} \times(1-x)}\right] \\
k=\frac{1}{2} n F A C \cdot \sqrt{D \cdot t}
\end{gathered}
$$

In this case, $Q$ represents the electrochemical charge flowing across the indicator electrodes of a coulometric sensor and $k$ is an electrochemical parameter related to redox molecule properties and the electrode reaction. $\Delta$ is a function of the potential difference applied to the sensor and $t$, the coulometric measurement time. Where eqn (1) is applied to peroxidase immunoassays measured via TMB redox processes, the $x$ term is a function of peroxidase kinetics; i.e. enzyme concentration and reaction time. For the peroxidase reaction time leading to the condition, $C_{\mathrm{TMB}_{\mathrm{red}}}=C_{\mathrm{TMB}_{\mathrm{ox}}},(x=0.5)$ the sensor response (sampled charge) takes the limiting form:

$$
Q_{\max }=k \cdot \frac{\sqrt{\Delta}-1}{\sqrt{\Delta}+1}
$$

Here, $Q_{\max }$ is the maximum electrochemical charge delivered by the sensor. The sensor response to blank samples is established by the surface binding characteristics of the peroxidase conjugate to the functionalised reaction chamber. High baseline readings are also observed with excessive peroxidase reaction times. Both factors strongly influence the coulometry leading to $Q \rightarrow Q_{\max }(x<0.5)$. The effect of this is to "compress" the charge readings for any set of samples or standards. In an extreme case, where $x>0.5$ (high conjugate adsorption or long peroxidase reaction time), the output of the sensor for a high analyte concentration can indeed fall below a sample of lower concentration, and could potentially be assigned as a low positive or even a negative.

\section{HSV-2 biochip and control samples}

The HerpeSelect ${ }^{\circledR} 2$ IgG ELISA kit from Focus Diagnostics is approved by the Food \& Drug Administration for clinical use and has been extensively studied and is widely available. ${ }^{19-23}$ The ELISA kit contains a number of control samples; these were tested repeatedly on the HSV-2 biochip to determine the reproducibility of the coulometric enzyme immunoassay. Three controls (negative, low positive and high positive) and the cut-off calibrator were each tested across 10 separate runs, with 2 biochips per sample per run and 2 sensors per biochip, yielding 40 measurements per sample in total. Results are summarised in Table 1 and demonstrated the HSV-2 biochip 
Table 1 HSV-2 biochip inter-assay reproducibility

\begin{tabular}{|c|c|c|c|c|c|}
\hline Sample & Mean charge $(\mu \mathrm{C})$ & SD & $\mathrm{CV}$ & Mean index value & $N$ \\
\hline HSV-2 cut-off calibrator & 0.855 & 0.151 & $17.7 \%$ & 1.00 & 40 \\
\hline HSV-2 negative control & 0.301 & 0.133 & $44.2 \%$ & 0.35 & 40 \\
\hline HSV-2 high positive control & 2.055 & 0.284 & $13.8 \%$ & 2.40 & 40 \\
\hline
\end{tabular}

$\mathrm{SD}$, standard deviation; CV, coefficient of variation.

assay was reproducible in terms of its qualitative performance. A representative coulometry trace for each HSV-2 control and calibrator is presented in Fig. 3.

\section{HSV-2 biochip and Focus HSV-2 ELISA on patient samples}

A total of 60 patient serum samples were each assayed on 3 separate HSV-2 biochips yielding 6 electrochemical charge measurement values per test sample. For each biochip assay run the cut-off calibrator was also tested in triplicate. Due to limitations in the manual processing of biochips, including provision of washing steps and maintaining consistent reagent incubation times across all devices, it was only possible to assay 5 samples during a given biochip assay run (i.e. 15 biochips tested in parallel). The index values for serum samples in the HSV-2 biochip dataset were each calculated from the mean electrochemical charge obtained for the cut-off calibrator sample during the same assay run. Where samples were tested at a non-standard dilution $(102 \times$ or $204 \times$ serum dilution), the sample index was multiplied by the fold-difference between test sample dilution and calibrator dilution (calibrator was consistently assayed at $51 \times$ dilution). The data obtained for the serum analysis is summarised in Table 2 .

All serum samples were additionally assayed by the Focus Diagnostics HerpeSelect® 2 IgG ELISA kit. These assays were run in accordance with the manufacturer's instructions. Each sample was run in duplicate and the entire sample panel was

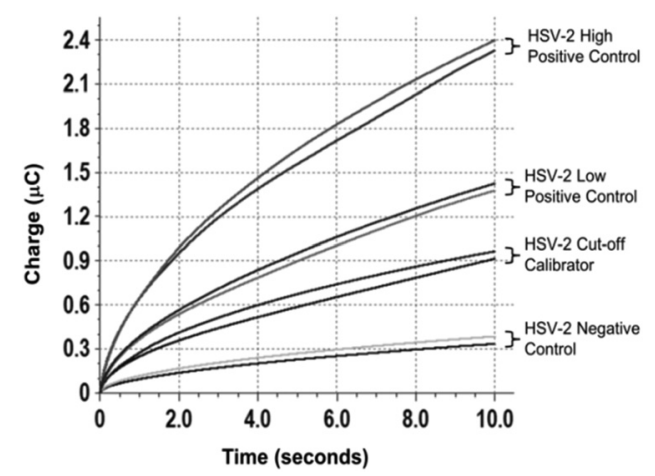

Fig. 3 HSV-2 control samples were tested as outlined in the materials and methods section. The charge-time plot above represents the sensor response from one HSV-2 biochip per sample. The two traces per sample type represent the electrochemical charge measurements from each sensor in a single biochip. The charge values used to calculate index values for patient samples in this study were all taken at the endpoint of the coulometric measurement (i.e. after 10 seconds). performed using two gG2-antigen coated microwell plates. ELISA HSV-2 index values for test serum samples were calculated relative to the cut-off calibrator sample from the same microwell plate. There was an excellent qualitative agreement between the results generated on the HSV-2 biochip assay and the supplied LIAISON® sample index values from Cerba Specimen Services. For the purposes of sensitivity and specificity determination, equivocal samples (index of $\geq 0.9$ to $<1.1$ ) were considered as negative for anti-HSV-2 antibodies. The sensitivity and specificity of the HSV-2 biochip test were both $100 \%$ using the LIAISON ${ }^{\circledR}$ assay as the reference method. Using the ELISA assay as the reference method, the sensitivity and specificity of the HSV-2 biochip assay was $96.8 \%$ and $100 \%$ respectively. These data demonstrate that the HSV-2 biochip assay performed to the same diagnostic standard as the FDA-approved assays, albeit on a small sample panel.

The sensitivity \& specificity of the biochip relative to the ELISA assay was independent of the sample dilution factor applied for the high index positive samples; these represent a subset of 8 samples of the 60 sera panel assessed $(13.3 \%$ of samples). The reported data for sensitivity and specificity includes all samples. Further, sensitivity and specificity relate to the correct classification of samples as being positive or negative for HSV-2 antibodies relative to a gold-standard method (in this study either the Focus or DiaSorin HSV-2 assays). The qualitative classification of a sample is determined by their response in the measuring system (biochip, ELISA or LIAISON) relative to the cut-off control assayed on the same plate (ELISA) or day (Biochip). Parallel assessment of the cut-off calibrator is required to minimise the impact of assay variances (e.g. sample incubation times, variations in dilutions, etc.). Whilst the HSV-2 assays, both biochip and ELISA, produce a continuous index score, they are ultimately qualitative in classifying samples as positive or negative, the magnitude of the index has no diagnostic significance.

The 8 'high index' sera identified by both Focus and DiaSorin test methods (index $>4$ ) yielded HSV-2 antibody positive index values when assayed at standard dilution factor of 51-fold. These index values ranged from 1.8 to 3.6 and are thus clearly positive for HSV-2. Therefore biochip sensitivity and specificity relative to the gold standard test methods are not impacted by the subsequent re-testing of these samples at increased sample dilution; this re-testing was performed solely to determine if the correlation between biochip and ELISA sample indices improved by increasing the measuring range of the biochip system. The linear correlation for non-corrected 


\begin{tabular}{|c|c|c|c|c|c|c|c|c|c|c|c|c|c|c|c|}
\hline Sample ID & Gender & $\begin{array}{l}\text { Age } \\
\text { (years) }\end{array}$ & Origin & $\begin{array}{l}\text { LIAISON } \\
\text { HSV-2 IgG } \\
\text { assay index }\end{array}$ & $\begin{array}{l}\text { HSV-2 } \\
\text { biochip } \\
\text { assay } \\
\text { sample } \\
\text { dilution }\end{array}$ & $\begin{array}{l}\text { Biochip } \\
\text { mean } \\
\text { charge } \\
(\mu \mathrm{C})\end{array}$ & $\begin{array}{l}\text { Biochip } \\
\text { SD }\end{array}$ & $\begin{array}{l}\text { Biochip } \\
\text { CV }\end{array}$ & $\begin{array}{l}\text { Biochip } \\
\text { cut-off } \\
\text { mean } \\
\text { charge }(\mu \mathrm{C})\end{array}$ & $\begin{array}{l}\text { Biochip } \\
\text { sample } \\
\text { index }^{a}\end{array}$ & 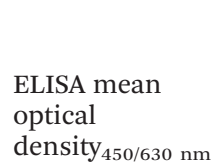 & $\begin{array}{l}\text { ELISA } \\
\text { SD }\end{array}$ & $\begin{array}{l}\text { ELISA } \\
\text { CV }\end{array}$ & 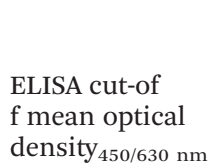 & $\begin{array}{l}\text { ELISA } \\
\text { sample } \\
\text { index }\end{array}$ \\
\hline 13 T0445114 & Male & 35 & Morocco & $<0.5$ & 51 & 0.288 & 0.097 & $33.7 \%$ & 0.790 & 0.4 & 0.103 & 0.004 & $3.4 \%$ & 0.304 & 0.3 \\
\hline 13 Т0464169 & Female & 19 & France & $<0.5$ & 51 & 0.342 & 0.048 & $14.2 \%$ & 0.648 & 0.5 & 0.030 & 0.001 & $4.7 \%$ & 0.304 & 0.1 \\
\hline $13 \mathrm{~T} 0466262$ & Female & 38 & Reunion Island & $<0.5$ & 51 & 0.283 & 0.122 & $43.0 \%$ & 0.648 & 0.4 & 0.021 & 0.001 & $3.4 \%$ & 0.304 & 0.1 \\
\hline $13 \mathrm{~T} 0470653$ & Male & 39 & France & $<0.5$ & 51 & 0.244 & 0.083 & $34.0 \%$ & 0.766 & 0.3 & 0.031 & 0.001 & $2.3 \%$ & 0.304 & 0.1 \\
\hline $13 \mathrm{~T} 0470927$ & Female & 48 & France & $<0.5$ & 51 & 0.728 & 0.087 & $11.9 \%$ & 0.766 & 1.0 & 0.197 & 0.004 & $1.8 \%$ & 0.304 & 0.6 \\
\hline $13 \mathrm{~T} 0475236$ & Female & 42 & France & $<0.5$ & 51 & 0.252 & 0.099 & $39.5 \%$ & 0.891 & 0.3 & 0.025 & 0.000 & $0.0 \%$ & 0.304 & 0.1 \\
\hline $13 \mathrm{~T} 0475390$ & Female & 30 & France & $<0.5$ & 51 & 0.542 & 0.161 & $29.7 \%$ & 0.891 & 0.6 & 0.046 & 0.001 & $3.1 \%$ & 0.304 & 0.2 \\
\hline 13 Т0480781 & Female & 44 & French Polynesia & $<0.5$ & 51 & 0.501 & 0.103 & $20.6 \%$ & 0.891 & 0.6 & 0.091 & 0.004 & $3.9 \%$ & 0.304 & 0.3 \\
\hline $13 \mathrm{~T} 0482332$ & Female & 31 & France & $<0.5$ & 51 & 0.068 & 0.032 & $47.6 \%$ & 0.376 & 0.2 & 0.061 & 0.008 & $12.9 \%$ & 0.304 & 0.2 \\
\hline 13 T0482367 & Female & 30 & Martinique & $<0.5$ & 51 & 0.032 & 0.023 & $72.6 \%$ & 0.376 & 0.1 & 0.051 & 0.009 & $18.2 \%$ & 0.304 & 0.2 \\
\hline 13 T0487266 & Male & 25 & France & $<0.5$ & 51 & 0.035 & 0.017 & $48.5 \%$ & 0.376 & 0.1 & 0.148 & 0.006 & $4.3 \%$ & 0.304 & 0.5 \\
\hline 13 T0490897 & Male & 51 & France & $<0.5$ & 51 & 0.388 & 0.105 & $26.9 \%$ & 0.648 & 0.6 & 0.020 & 0.001 & $7.1 \%$ & 0.298 & 0.1 \\
\hline 13 Т0491281 & Female & 16 & France & $<0.5$ & 51 & 0.190 & 0.077 & $40.6 \%$ & 0.790 & 0.2 & 0.056 & 0.001 & $1.3 \%$ & 0.298 & 0.2 \\
\hline 13 Т0496713 & Female & 44 & France & $<0.5$ & 51 & 0.217 & 0.087 & $40.3 \%$ & 0.626 & 0.3 & 0.034 & 0.005 & $14.8 \%$ & 0.298 & 0.1 \\
\hline 13 T0498896 & Female & 57 & France & $<0.5$ & 51 & 0.417 & 0.126 & $30.3 \%$ & 0.648 & 0.6 & 0.197 & 0.018 & $9.3 \%$ & 0.298 & 0.7 \\
\hline 13 T0500895 & Male & 32 & France & $<0.5$ & 51 & 0.504 & 0.051 & $10.2 \%$ & 0.626 & 0.8 & 0.117 & 0.001 & $1.2 \%$ & 0.298 & 0.4 \\
\hline 13 T0506737 & Male & 83 & France & $<0.5$ & 51 & 0.253 & 0.072 & $28.5 \%$ & 0.626 & 0.4 & 0.081 & 0.000 & $0.0 \%$ & 0.298 & 0.3 \\
\hline 13 T0507029 & Male & 85 & France & $<0.5$ & 51 & 0.276 & 0.130 & $47.0 \%$ & 0.710 & 0.4 & 0.031 & 0.001 & $2.3 \%$ & 0.298 & 0.1 \\
\hline 13 T0509304 & Female & 21 & France & $<0.5$ & 51 & 0.547 & 0.116 & $21.2 \%$ & 0.710 & 0.8 & 0.086 & 0.002 & $2.5 \%$ & 0.298 & 0.3 \\
\hline 13 Т0511323 & Male & 35 & France & $<0.5$ & 51 & 0.330 & 0.091 & $27.6 \%$ & 0.710 & 0.5 & 0.050 & 0.001 & $2.8 \%$ & 0.298 & 0.2 \\
\hline 13 T0511392 & Male & 35 & France & $<0.5$ & 51 & 0.481 & 0.206 & $42.8 \%$ & 1.091 & 0.4 & 0.044 & 0.005 & $11.4 \%$ & 0.298 & 0.1 \\
\hline 13 T0513104 & Male & 23 & France & $<0.5$ & 51 & 0.310 & 0.047 & $15.0 \%$ & 0.790 & 0.4 & 0.060 & 0.004 & $7.1 \%$ & 0.298 & 0.2 \\
\hline $13 \mathrm{~T} 0522750$ & Female & 23 & France & $<0.5$ & 51 & 0.905 & 0.080 & $8.8 \%$ & 1.091 & 0.8 & 0.109 & 0.005 & $4.6 \%$ & 0.298 & 0.4 \\
\hline 13 T0527146 & Female & 26 & France & $<0.5$ & 51 & 0.227 & 0.046 & $20.4 \%$ & 0.554 & 0.4 & 0.038 & 0.001 & $1.9 \%$ & 0.298 & 0.1 \\
\hline 13 T0527211 & Male & 33 & France & $<0.5$ & 51 & 0.214 & 0.122 & $57.0 \%$ & 0.554 & 0.4 & 0.075 & 0.000 & $0.0 \%$ & 0.298 & 0.3 \\
\hline 13 T0529135 & Female & 24 & France & $<0.5$ & 51 & 0.277 & 0.092 & $33.2 \%$ & 0.554 & 0.5 & 0.023 & 0.001 & $3.1 \%$ & 0.298 & 0.1 \\
\hline 13 T0537549 & Male & 15 & France & $<0.5$ & 51 & 0.706 & 0.152 & $21.6 \%$ & 1.040 & 0.7 & 0.197 & 0.007 & $3.6 \%$ & 0.298 & 0.7 \\
\hline 13 Т0549351 & Female & 40 & France & $<0.5$ & 51 & 0.508 & 0.117 & $23.0 \%$ & 0.946 & 0.5 & 0.056 & 0.004 & $7.6 \%$ & 0.298 & 0.2 \\
\hline 14 D0391962 & Male & 64 & France & 0.7 & 51 & 0.492 & 0.125 & $25.4 \%$ & 0.604 & 0.8 & 0.475 & 0.011 & $2.2 \%$ & 0.298 & 1.6 \\
\hline 13 T0530393 & Male & 78 & France & 1.0 & 51 & 0.783 & 0.243 & $31.1 \%$ & 0.790 & 1.0 & 0.041 & 0.001 & $3.4 \%$ & 0.298 & 0.1 \\
\hline $13 \mathrm{~T} 0470933$ & Male & 38 & France & 1.2 & 51 & 1.228 & 0.198 & $16.1 \%$ & 0.766 & 1.6 & 0.975 & 0.017 & $1.7 \%$ & 0.304 & 3.2 \\
\hline $13 \mathrm{~T} 0434783$ & Female & 24 & Mayotte & 1.4 & 51 & 1.471 & 0.112 & $7.6 \%$ & 0.946 & 1.6 & 1.031 & 0.012 & $1.2 \%$ & 0.304 & 3.4 \\
\hline $13 \mathrm{~T} 0517588$ & Female & 37 & France & 1.5 & 51 & 1.395 & 0.254 & $18.2 \%$ & 1.040 & 1.3 & 0.957 & 0.023 & $2.4 \%$ & 0.298 & 3.2 \\
\hline 13 T0528664 & Female & 23 & France & 1.5 & 51 & 2.140 & 0.307 & $14.3 \%$ & 0.946 & 2.3 & 1.601 & 0.025 & $1.6 \%$ & 0.298 & 5.4 \\
\hline 13 Т0565931 & Male & 62 & France & 1.6 & 51 & 0.883 & 0.055 & $6.2 \%$ & 0.604 & 1.5 & 1.183 & 0.018 & $1.6 \%$ & 0.298 & 4.0 \\
\hline 13 T0395081 & Female & 64 & France & 1.7 & 51 & 1.985 & 0.146 & $7.4 \%$ & 1.040 & 1.9 & 1.091 & 0.013 & $1.2 \%$ & 0.304 & 3.6 \\
\hline 13 T0065482 & Female & 43 & France & 1.8 & 51 & 1.343 & 0.096 & $7.1 \%$ & 0.891 & 1.5 & 0.990 & 0.016 & $1.6 \%$ & 0.304 & 3.3 \\
\hline 13 Т0086841 & Male & 55 & Mayotte & 1.8 & 51 & 0.845 & 0.134 & $15.8 \%$ & 0.376 & 2.2 & 1.174 & 0.004 & $0.3 \%$ & 0.304 & 3.9 \\
\hline $13 \mathrm{~T} 0087037$ & Male & 55 & Mayotte & 1.8 & 51 & 2.187 & 0.297 & $13.6 \%$ & 0.648 & 3.4 & 1.248 & 0.037 & $3.0 \%$ & 0.304 & 4.1 \\
\hline 13 Т0103221 & Female & 48 & France & 1.8 & 51 & 1.638 & 0.235 & $14.4 \%$ & 0.626 & 2.6 & 1.688 & 0.042 & $2.5 \%$ & 0.304 & 5.6 \\
\hline 13 Т0123866 & Female & 30 & France & 1.8 & 51 & 0.985 & 0.050 & $5.1 \%$ & 0.710 & 1.4 & 1.377 & 0.050 & $3.6 \%$ & 0.304 & 4.5 \\
\hline 13 Т0162515 & Male & 70 & Martinique & 1.8 & 51 & 2.031 & 0.310 & $15.3 \%$ & 1.091 & 1.9 & 1.261 & 0.024 & $1.9 \%$ & 0.304 & 4.1 \\
\hline 13 Т0167364 & Female & 43 & French Guiana & 1.8 & 51 & 1.148 & 0.130 & $11.3 \%$ & 0.554 & 2.1 & 1.021 & 0.006 & $0.6 \%$ & 0.304 & 3.4 \\
\hline $13 \mathrm{~T} 0022372$ & Female & 52 & France & 1.9 & 51 & 1.877 & 0.147 & $7.8 \%$ & 0.766 & 2.4 & 1.286 & 0.011 & $0.8 \%$ & 0.304 & 4.2 \\
\hline 13 T0026255 & Female & 41 & Madagascar & 1.9 & 51 & 1.133 & 0.156 & $13.8 \%$ & 0.648 & 1.7 & 0.881 & 0.023 & $2.7 \%$ & 0.304 & 2.9 \\
\hline 13 T0033959 & Female & 32 & France & 2.5 & 51 & 1.882 & 0.295 & $15.7 \%$ & 0.766 & 2.5 & 1.300 & 0.062 & $4.8 \%$ & 0.304 & 4.3 \\
\hline 13 T0140910 & Female & 60 & France & 2.7 & 51 & 1.926 & 0.212 & $11.0 \%$ & 0.554 & 3.5 & 1.977 & 0.077 & $3.9 \%$ & 0.304 & 6.5 \\
\hline
\end{tabular}




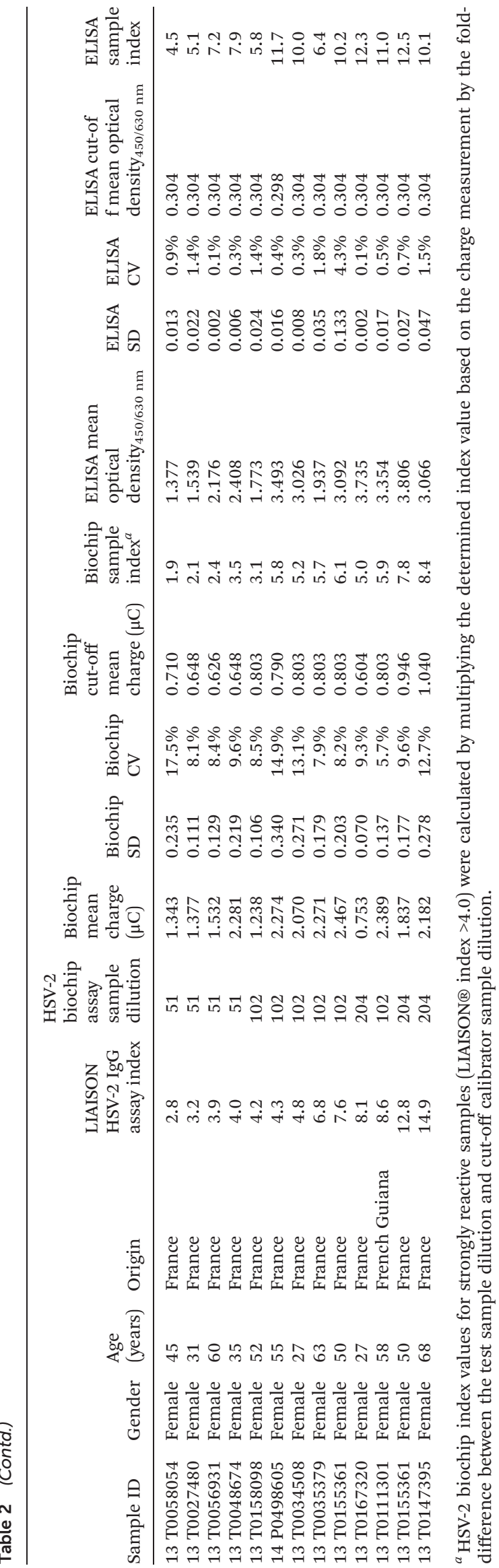

biochip index values is lower $\left(r^{2} 0.36\right.$ or 0.71$)$ versus correcting index values for sample dilution. This reflected the upper limit of charge detection in the HSV-2 biochip system.

Owing to the biochip geometry, the maximum electrochemical charge observed in the HSV-2 pilot trials to optimise the assay design was $<3.0 \mu \mathrm{C}$. Using simple linear regression analysis, the coefficient of determination of the HSV-2 biochip sample index values with sample index values from the two reference methods was poor $\left(r^{2} 0.3005\right.$ or 0.6237). Nevertheless, the HSV-2 biochip sensitivity and specificity in relation to both reference methods remained unaffected. The reason for the poor linear relationship between datasets was that the charge measurements for high positive samples did not truly reflect the intense peroxidase activity resulting from these samples. In order to account for the limitation in electrochemical charge generation on the HSV-2 biochip, samples that were strongly reactive on the LIAISON ${ }^{8}$ system (index values $>4.0$ ) were re-assayed on the HSV-2 biochip at increased sample dilution and adjusting the sample index values accordingly. Scatter plots of HSV-2 biochip index values plotted against the LIAISON ${ }^{\circ}$ HSV-2 index values (Fig. 4) and Focus HerpeSelect ${ }^{2} 2$ IgG index values (Fig. 5) are shown. Adjusting

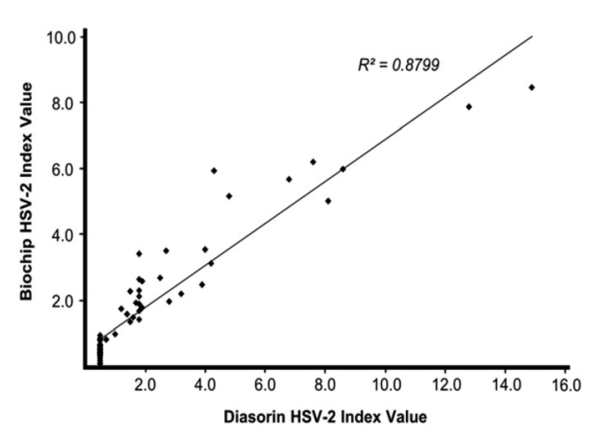

Fig. 4 Sample index values on the DiaSorin HSV-2 LIAISON® assay are plotted ( $x$-axis) against the index values on the HSV-2 biochip assay ( $y$-axis). The linear-fit trendlines and coefficients of determination are shown.

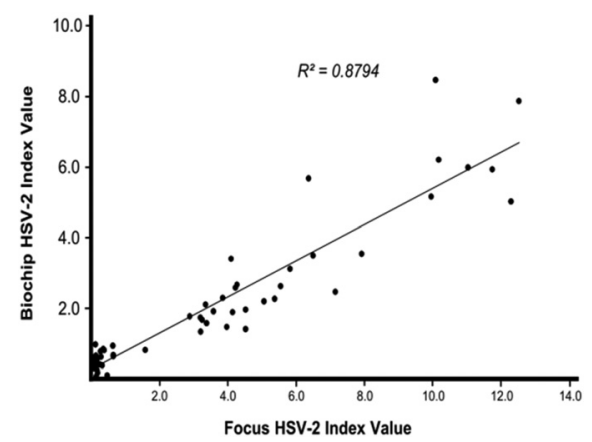

Fig. 5 Sample index values on the HerpeSelect ${ }^{\circledR} 2$ IgG assay are plotted ( $x$-axis) against the index values on the HSV-2 biochip assay ( $y$-axis). The linear-fit trendlines and coefficients of determination are shown. 
the high positive sample index values by increasing sample dilution greatly increased the coefficient of determination between the HSV-2 biochip results and the two reference assay methods ( $r^{2} 0.8799$ or 0.8794$)$. These data demonstrate that the HSV-2 biochip assay performance closely mirrors the chosen in vitro diagnostic reference tests.

The poor correlation observed with respect to the full dataset, specifically the sensor response to very high positives for the 51-fold dilution data, is to a very great degree a direct consequence of the non-linear charge-concentration characteristic of the coulometric sensors. This means in practice it is possible in cases of extremely high positives to obtain an output from the sensors lower than less concentrated (HSV-2 antibody) samples. Rescaling the sensor for these high samples by dilution is therefore effectively decreasing the peroxidase activity and altering the concentrations of $\mathrm{TMB}_{\mathrm{ox}}$ and $\mathrm{TMB}_{\text {red }}$, upon which the electrodes of each sensor respond.

The generic membrane-touch biochip featuring an immunochemical reaction chamber, a hydraulic microfluidics pump and coulometric sensors was assessed for the qualitative determination of antibodies against HSV-2 in human serum. The assay comprises standard immunoassay reagents with a high activity recombinant gG2 antigen and electrochemical charge measurement. In terms of diagnostic performance, the HSV-2 biochip closely matched two FDA cleared HSV-2 serological diagnostic assays. The manufacturers of the chosen reference assay methods, namely DiaSorin and Focus Diagnostics, have both performed extensive assay validation as part of the IVD approval process. Both tests were validated against a gold standard HSV-2 Western blot method and in various populations have high sensitivities $(94.8 \%-100 \%)$ and specificities (96.1\%-100\%) detailed in the assay product inserts. In fact, a comparative study between the Focus and DiaSorin HSV-2 tests revealed a $100 \%$ agreement in a panel of 247 samples from STD clinic patients and expectant mothers. ${ }^{27}$ The HSV-2 biochip assay has high sensitivity $(96.8 \%-100 \%)$ and specificity $(100 \%)$ compared to these two reference assay methods. Therefore, by extension, the HSV-2 biochip assay is very likely to perform to a comparable standard against the HSV-2 serological gold standard Western blot method. The excellent correlation of the HSV-2 biochip is likely due to the use of a high activity recombinant HSV-2 gG2 antigen.

Further to the qualitative correlation of the HSV-2 biochip with the two reference assays, there was also a good semiquantitative relationship. This is not unexpected since the biochip's coulometric sensors are quantitative for peroxidase activity. Using index values as a comparator, the HSV-2 biochip yielded a high linear correlation with the LIAISON ${ }^{\circ}$ test $\left(r^{2}\right.$ $0.8799)$ and with the ELISA test $\left(r^{2} 0.8794\right)$. In fact the correlation of the HSV-2 biochip dataset with the two reference assays was better than the linear correlation of the reference assays with one another $\left(r^{2}\right.$ 0.732). This indicates biochip could be adapted to perform quantitative assays as well as have broader utility for other diagnostic targets.

The HSV-2 biochip study has several limitations, which should be viewed in the context of an early phase technical development to bring a disruptive point-of-care technology to market. Firstly, the human panel used was small (60 sera) and collected from a narrow geographic range, originating predominantly in France. Secondly, only limited demographic data was available for the selected samples, namely gender and age of patients. Certain factors that are known to impact the accuracy of serological HSV-2 tests, such as decreased specificity in populations from sub-Saharan Africa or in HIV-positive patients may or may not have played a role in this study. ${ }^{23}$ However, as such factors are known to impact on the analytical performance of other more established HSV-2 serological diagnostic tests, this bias would not be specific to the HSV-2 biochip test.

The rapid HSV-2 tests referenced in this work have typical assay times of between 5 and 15 minutes. By contrast the HSV-2 biochip, was optimised using well-characterised commercial immuno-reagents for a 65 minutes assay. This compares favourably to the Focus Diagnostics HerpeSelect ${ }^{\circledR} 2$ ELISA IgG assay of 100 minutes. There is ample scope to reduce the assay time of the HSV-2 biochip by reconfiguring the chip's microfluidics and coulometric sensor architecture as well as other assay protocol optimisations.

\section{Conclusion}

A generation of physical chemists and biosensor developers have immobilised antibodies directly onto electrodes with varying degrees of success; often demonstrating poor analytical performance. The use of a shunt-fluidic mechanism actuated by a simple hydraulic pump (membrane touch) allows immunoassay reactions to proceed in isolation from the indicator electrodes of a coulometric sensor. This reduces significantly non-specific binding and background signals. In our laboratory no matter what blocking reagent strategy was attempted to printed carbon surfaces, background readings associated with non-specific adsorptions were consistently more than five-fold larger than any protein-blocked polymer surface.

The membrane-touch biochip is in its early development and the authors emphasise the many challenging technical issues to address before a HSV-2 biochip can become a meaningful out-of-laboratory ELISA technology. The device presented relies on manual sample delivery and reagent injection and so the chip would require an external fluidic device to complete these functions. The on-chip pump was controlled manually and would require an external electromechanical device to actuate its operation. The sensors of the biochip offer a quantitative measure over that of assigning only positive highs and lows or negatives to blood samples (qualitative), as shown in this work. In order for HSV-2 biochip to fulfil its promise the device would require extensive calibration for qualitative and quantitative applications against approved laboratory methods.

The application of a membrane-touch biochip to the qualitative HSV-2 immunoassay for human samples was demon- 
strated in this work. Assay performance of a novel microfluidic coulometric sensor responsive to HSV-2 antibodies was validated against diagnostics industry standard methods. The results from a human panel confirm the potential of the biochip to fulfil an unmet need for rapid, accurate and cost effective clinical diagnosis and management of HSV-2 infection. Adaptation of this coulometric technology to diagnostic assays for emerging infectious diseases is now under consideration by the project team.

\section{Acknowledgements}

MiCRA Biodiagnostics kindly acknowledge the assistance of the staff at the Haematology Laboratory, Department of Laboratory Medicine, Tallaght Hospital (AMNCH). A special thank you to Dympna Murphy, Chief Medical Scientist at the Haematology Laboratory for the coordination of the work leading to the human panel testing of the HSV-2 Biochip. The authors wish to thank Prof. Rhoda A. Morrow, Head, Faculty Affairs, Vaccine and Infectious Disease Division, University of Washington for guidance on the preparation of the manuscript. The HSV-2 Biochip study was funded by Enterprise Ireland through the Innovation Partnership Programme, Express Scheme. The project was co-funded by Aalto Bio Reagents Ltd (Grant Number: IP 20150529E).

\section{Notes and references}

1 R. Gupta, T. Warren and A. Wald, Lancet, 2007, 370, 2127.

2 D. I. Bernstein, A. R. Bellamy, E. W. Hook 3rd, M. J. Levin, A. Wald, M. G. Ewell, P. A. Wolff, C. D. Deal, T. C. Heineman, G. Dubin and R. B. Belshe, Clin. Infect. Dis., 2013, 56, 344.

3 K. J. Looker, G. P. Garnett and G. P. Schmid, Bull. W. H. O., 2008, 86, 805.

4 Centers for Disease Control and Prevention (CDC), $M M W R$ Morb. Mortal. Wkly. Rep., 2010, 59, 456.

5 G. Paz-Bailey, M. Ramaswamy, S. J. Hawkes and A. M. Geretti, Sex. Transm. Infect., 2007, 83, 16.

6 C. Johnston, D. M. Koelle and A. Wald, J. Clin. Invest., 2011, 121, 4600.

7 A. G. Langenberg, L. Corey, R. L. Ashley, W. P. Leong and S. E. Straus, N. Engl. J. Med., 1999, 341, 1432.
8 E. E. Freeman, H. A. Weiss, J. R. Glynn, P. L. Cross, J. A. Whitworth and R. J. Hayes, AIDS, 2006, 20, 73.

9 A. Wald, J. Zeh, S. Selke, T. Warren, A. J. Ryncarx, R. Ashley, J. N. Krieger and L. Corey, N. Engl. J. Med., 2000, 342, 844.

10 D. T. Fleming, G. M. McQuillan, R. E. Johnson, A. J. Nahmias, S. O. Aral, F. K. Lee and M. E. St Louis, N. Engl. J. Med., 1997, 337, 1105.

11 P. Leone, D. T. Fleming, A. W. Gilsenan, L. Li and S. Justus, Sex. Transm. Dis., 2004, 31, 311.

12 G. J. Mertz, J. Benedetti, R. Ashley, S. A. Selke and L. Corey, Ann. Intern. Med., 1992, 116, 197.

13 J. LeGoff, H. Péré and L. Bélec, J. Virol., 2014, 11, 83.

14 A. Dolan, F. E. Jamieson, C. Cunningham, B. C. Barnett and D. J. McGeoch, J. Virol., 1998, 72, 2010.

15 F. K. Lee, R. M. Coleman, L. Pereira, P. D. Bailey, M. Tatsuno and A. J. Nahmias, J. Clin. Microbiol., 1985, 22, 641.

16 R. L. Ashley, J. Militoni, F. Lee, A. Nahmias and L. Corey, J. Clin. Microbiol., 1988, 26, 662.

17 D. L. Parkes, C. M. Smith, J. M. Rose, J. Brandis and S. R. Coates, J. Clin. Microbiol., 1991, 29, 778.

18 R. L. Ashley, Sex. Transm. Infect., 2001, 77, 232.

19 H. E. Prince, C. E. Ernst and W. R. Hogrefe, J. Clin. Lab. Anal., 2000, 14, 13.

20 R. A. Morrow, D. Friedrich and E. Krantz, J. Clin. Microbiol., 2003, 41, 5212.

21 E. van Dyck, A. Buvé, H. A. Weiss, J. R. Glynn, D. W. Brown, B. De Deken, J. Parry and R. J. Hayes, J. Clin. Microbiol., 2004, 42, 2961.

22 J. LeGoff, P. Mayaud, G. Gresenguet, H. A. Weiss, K. Nzambi, E. Frost, J. Pepin and L. Belec, J. Clin. Microbiol., 2008, 46, 1914.

23 S. Biraro, P. Mayaud, R. A. Morrow, H. Grosskurth and H. A. Weiss, Sex. Transm. Dis., 2011, 38, 140.

24 B. Seddon, R. O’Reilly, E. Dempsey, J. Hayes and B. Singh, Micro-reagent handler and cartridge assembly, European Patent Office PCT/EP2013/073876, 2013.

25 P. D. Josephy, T. Eling and R. P. Mason, J. Biol. Chem., 1982, 257, 3669.

26 H. L. Kies, Thesis, Theorie en toepassingen van titraties volgens de methode van het dead-stop eindpunt, Delft University of Technology, December 1956.

27 A. W. Maters, C. V. Wright, M. T. Lee, G. Schwichtenberg and B. Detrick, Diagn. Microbiol. Infect. Dis., 2012, 73, 273. 